\title{
OSCILLATORY BEHAVIOR OF THIRD ORDER DIFFERENTIAL EQUATIONS
}

\author{
GARY D. JONES
}

\begin{abstract}
It is shown that if $p(x) \leqq 0, q(x)>0$ and if $y^{\prime \prime}+$ $p y^{\prime}+q y=0$ has an oscillatory solution then every nonoscillatory solution is a constant multiple of one nonoscillatory solution.
\end{abstract}

A solution of

$$
y^{\prime \prime \prime}+p(x) y^{\prime}+q(x) y=0
$$

will be said to be oscillatory if it changes signs for arbitrarily large values of $x$. Other solutions will be said to be nonoscillatory. It will be assumed that $p(x), q(x)$, and $p^{\prime}(x)$ are continuous on $[0,+\infty)$.

The first theorem will be in the setting of Class I or Class II equations as defined by Hanan [3].

THEOREM 1. Suppose (1) is of Class I or Class II. If (1) has an oscillatory solution and if $N$ is a nontrivial nonoscillatory solution of its adjoint

$$
y^{\prime \prime \prime}+p(x) y^{\prime}+\left(p^{\prime}(x)-q(x)\right) y=0
$$

then there are two independent oscillatory solutions of (1) that satisfy

$$
\left(\frac{y^{\prime}}{N}\right)^{\prime}+\left(\frac{N^{\prime \prime}+p N}{N^{2}}\right) y=0 .
$$

Proof. Since (1) is of Class I or Class II, so is (2) [3]. Thus, if $N$ is a nontrivial nonoscillatory solution of (2) there is an $a \in[0,+\infty)$ such that $N(x) \neq 0$ for $x>a$. Further, since (1) has an oscillatory solution, there are two independent oscillatory solutions $y_{1}$ and $y_{2}$ of (2) [5]. It is easily verified that $y_{1} N^{\prime}-N y_{1}^{\prime}$ and $y_{2} N^{\prime}-N y_{2}^{\prime}$ are independent oscillatory solutions of (1) and (3).

Presented to the Society, September 3, 1971 under the title Oscillation properties of third order differential equations; received by the editors March 19, 1973 and, in revised form, June 26, 1973.

AMS (MOS) subject classifications (1970). Primary 34A30, 34C10.

Key words and phrases. Differential equations, third order, oscillation, basis of solutions.

(c) American Mathematical Society 1974 
Corollary. Suppose (1) is of Class I or II and has an oscillatory solution. If $N$ and $y$ are independent solutions of (2) such that $N$ is nonoscillatory, then $N y^{\prime}-y N^{\prime}$ is an oscillatory solution of (1).

PROOF. Under the conditions of the Corollary (3) is oscillatory and $N y^{\prime}-y N^{\prime}$ is a solution of (3).

The proof of the following theorem is essentially contained in the proof Theorem 1.5 [6], but is included here for completeness.

Theorem 2. Suppose $p(x) \leqq 0, q(x)>0$ and that (1) has an oscillatory solution. Suppose $N(x)$ is a solution of (2) defined by $N(a)=N^{\prime}(a)=0$, $N^{\prime \prime}(a)=1$ for $a \in[0,+\infty)$. Then $N(x)>0, N^{\prime}(x)>0$ and $N^{\prime \prime}(x)>N^{\prime \prime}(x)+$ $p(x) N(x)>1$ for $x>a$.

Proof. By [6], (1) is Class I. Thus (2) is Class II [3]. It follows that $N(x)>0$ for $x>a$. Now $\left(N^{\prime \prime}(x)+p(x) N(x)\right)^{\prime}=q(x) N(x)>0$ for $x>a$. Thus since $N^{\prime \prime}+p N$ is an increasing function of $x$ for $x>a$ and since $p(x) \leqq 0, \quad N^{\prime \prime}(x)>N^{\prime \prime}(x)+p(x) N(x)>N^{\prime \prime}(a)+p(a) N(a)=1$ for $x>a$. It now follows that $N^{\prime}(x)>0$ for all $x>a$.

Theorem 3. Suppose (1) is Class I or II, that $q(x)>0$ and that (2) has a nonoscillatory solution $N$ such that $N(x)>0$ and $N^{\prime}(x)>0$ for $x>a$. Then

$$
G[y(x)] \equiv N y^{\prime 2}+\left(N^{\prime \prime}+p N\right) y^{2}
$$

is an increasing function of $x$ for $x>a$, where $y$ is any solution of (3).

Proof.

$$
\begin{aligned}
G^{\prime}[y(x)] & =2 N y^{\prime} y^{\prime \prime}+N^{\prime} y^{\prime 2}+2 y\left(N^{\prime \prime}+p N\right) y^{\prime}+q N y^{2} \\
& =2 y^{\prime}\left[N^{\prime} y^{\prime}-\left(N^{\prime \prime}+p N\right) y\right]+N^{\prime} y^{\prime 2}+2 y y^{\prime}\left(N^{\prime \prime}+p N\right)+q N y^{2} \\
& =3 N^{\prime} y^{\prime 2}+q N y^{2}>0 \text { for } x>a .
\end{aligned}
$$

Thus, the result follows.

Our main result which generalizes results of Lazer [6] and Gregus [2] now follows.

THEOREM 4. If $p(x) \leqq 0, q(x)>0$ and (1) has an oscillatory solution then every nonoscillatory solution is a constant multiple of one nonoscillatory solution.

Proof. Let $N$ be a solution of (2) defined by $N(a)=N^{\prime}(a)=0, N^{\prime \prime}(a)=1$ for $a \in[0,+\infty)$. Since $p(x) \leqq 0$ and $q(x)>0,(1)$ is Class $I$ and has a solution $z(x)$ such that $z(x)>0, z^{\prime}(x)<0, z^{\prime \prime}(x)>0$ for all $x \in[0,+\infty)$ [6]. Let $u_{1}$ and $u_{2}$ be independent solutions of (1) that satisfy (3). Then $z, u_{1}$, and $u_{2}$ is a basis for the solution space of (1). Assuming that there 
are two independent solutions of (1) that are nonoscillatory then $z+$ $c_{1} u_{1}+c_{2} u_{2}$ is a nonoscillatory solution of (1) for some $c_{1}$ and $c_{2}$ not both zero. Let $-y_{1}=c_{1} u_{1}+c_{2} u_{2}$ and let $y_{2}$ be from the space spanned by $\left\{u_{1}, u_{2}\right\}$ independent from $y_{1}$. By [6], $\left|z(x)-y_{1}(x)\right|>0$. Since $y_{1}$ is oscillatory and $z(x)>0$ it is clear that $z(x)-y_{1}(x)>0$ for $x \in[0,+\infty)$.

Since $y_{1}, y_{2}, z$ are independent solutions of (1)

where $k$ is a constant.

$$
0 \neq k=\left|\begin{array}{ccc}
y_{1} & y_{2} & z \\
y_{1}^{\prime} & y_{2}^{\prime} & z^{\prime} \\
y_{1}^{\prime \prime} & y_{2}^{\prime \prime} & z^{\prime \prime}
\end{array}\right|
$$

Expanding, we obtain

$$
z\left[N^{\prime \prime}+p N\right]-z^{\prime} N^{\prime}+z^{\prime \prime} N=k_{1} \neq 0
$$

where $k_{1}$ is a constant. By the observation about $z$ noted above and Theorem 2, $z\left[N^{\prime \prime}+p N\right],-z^{\prime} N^{\prime}$ and $z^{\prime \prime} N$ are each positive for $x>a$. Thus $0<z\left[N^{\prime \prime}+p N\right]<k_{1}$ for $x>a$. Let $\left\{x_{n}\right\}_{n=1}^{\infty}$ be a sequence such that $y_{1}^{\prime}\left(x_{n}\right)=0$ and $y_{1}^{\prime \prime}\left(x_{n}\right)<0$ such that $x_{n} \rightarrow \infty$. Then

$$
\begin{aligned}
k_{1} & >z\left(x_{n}\right)\left[N^{\prime \prime}\left(x_{n}\right)+p\left(x_{n}\right) N\left(x_{n}\right)\right] \\
& \geqq y_{1}\left(x_{n}\right)\left[N^{\prime \prime}\left(x_{n}\right)+p\left(x_{n}\right) N\left(x_{n}\right)\right]>0 .
\end{aligned}
$$

But, by [4], $\lim _{x \rightarrow \infty} z(x)=0$. Therefore $y_{1}^{2}\left(x_{n}\right)\left[N^{\prime \prime}\left(x_{n}\right)+p\left(x_{n}\right) N\left(x_{n}\right)\right] \rightarrow 0$ as $n \rightarrow \infty$. But this is not possible since $G\left[y_{1}(x)\right]$ in Theorem 3 is increasing.

The following result gives a condition for certain equations of Class II to have behavior similar to that observed by Ahmad and Lazer in [1].

THEOREM 5. If $p(x) \leqq 0, q(x)-p^{\prime}(x)<0$ and (1) has an oscillatory solution, then there exist two linearly independent oscillatory solutions of (1) whose zeros separate and such that a solution of (1) is oscillatory if and only if it is a nontrivial linear combination of them.

Proof. Since $p(x) \leqq 0$ and $p^{\prime}(x)-q(x)>0$, there is a solution $N$ of (2) such that $N(x)>0$ for all $x \in[0,+\infty)$ [6]. Thus by Theorem 1 there are two linearly independent oscillatory solutions, $y_{1}$ and $y_{2}$, of (1) whose zeros separate.

Suppose there is an oscillatory solution of (1) that is not a linear combination of $y_{1}$ and $y_{2}$. Then by [5] there are two independent nonoscillatory solutions of (2), but this is contrary to Theorem 4 .

\section{REFERENCES}

1. Shair Ahmad and A. C. Lazar, On the oscillatory behavior of a class of linear third order differential equations, J. Math. Anal. Appl. 28 (1970), 681-689. MR 40 \#1646. 
2. M. Gregus, Uber einige Eigenschaften der Lösungen der Differentialgliechungen $y^{\prime \prime \prime}+2 A y^{\prime}+\left(A^{\prime}+b\right) y=0, A \leqq 0$, Czechoslovak Math. J. 11 (86)(1961), 106-114. MR 24 \#A882.

3. M. Hanan, Oscillation criteria for third-order linear differential equations, Pacific J. Math. 11 (1961), 919-944. MR 26 \#2695.

4. G. Jones, An asymptotic property of solutions of $y^{\prime \prime}+p y^{\prime}+q y=0$, Pacific J. Math. 47 (1973), 135-138.

5. — Oscillation properties of third order differential equations, Rocky Mt. J. Math. 3 (1973), 507-513.

6. A. C. Lazer, The behavior of solutions of the differential equation $y^{\prime \prime}+p(x) y^{\prime}+$ $q(x) y=0$, Pacific J. Math. 17 (1966), 435-466. MR 33 \#1552.

Department of Mathematics, Murray State University, Murray, Kentucky 42071 